\title{
A two-component small multidrug resistance pump
functions as a metabolic valve during nicotine
catabolism by Arthrobacter nicotinovorans \\ Correspondence \\ Roderich Brandsch \\ roderich.brandsch@biochemie. \\ uni-freiburg.de \\ Received 9 November 2006 \\ Revised 8 January 2007 \\ Accepted 10 January 2007

\author{
Petra Ganas, ${ }^{1}$ Marius Mihasan, ${ }^{1,3}$ Gabor L. Igloi ${ }^{2}$ and Roderich Brandsch ${ }^{1}$ \\ ${ }^{1}$ Institute of Biochemistry and Molecular Biology, Centre for Biochemistry and Molecular Cell \\ Research, Albrecht-Ludwigs University, Freiburg, Germany \\ ${ }^{2}$ Institute of Biology III, Albrecht-Ludwigs University, Freiburg, Germany \\ ${ }^{3}$ Department of Biochemistry, Alexandru-loan-Cuza University, lasi, Romania
}

\section{INTRODUCTION}

L-Nicotine, the main tobacco alkaloid, besides being highly toxic, represents a serious health problem by leading to addiction in smokers. Despite the enormous amount of research on its pharmacological effects in humans and in animal models, very little is known about the cellular transport mechanisms for nicotine. It is generally considered to diffuse easily through the skin (Hukkanen et al., 2005). Nicotine derivatives, but not nicotine itself, apparently crosses the blood-brain barrier by a general transporter of cationic drugs (Lee et al., 2001). On the other hand, nicotine is utilized as a growth substrate by certain bacteria and these play an important role in the detoxification of tobacco waste by mineralizing this heterocyclic aromatic compound. A detailed knowledge of the pathways of nicotine degradation, of the participating enzymes and of its genetic organization is of biotechnological relevance (Brandsch, 2006).

Abbreviations: $\mathrm{AO}$, amine oxidase; CCCP, carbonyl cyanide $m$ chlorophenylhydrazone; 2,6-DHPON, 2,6-dihydroxypseudooxynicotine; 2,6-DHP, 2,6-dihydroxypyridine; 6-HLN, 6-hydroxy-L-nicotine; Kn, kanamycin; LB, lysogeny broth; MABO, $\gamma$-N-methylaminobutyrate oxidase; PMF, proton-motive force; PONH, pseudooxynicotine hydrolase; SMR, small multidrug resistance; TPP ${ }^{+}$, tetraphenylphosphonium.
As nicotine is a strong base, with a $\mathrm{p} K_{\mathrm{a}}$ above 11 for the tertiary amine of the pyrrolidine ring, diffusion-mediated crossing of the cell membrane by this cation may be limited. Micro-organisms have evolved specific uptake systems, including transporters for highly polar compounds and aromatics (Leveau et al., 1998; Locher et al., 1993; Nichols \& Harwood, 1997; Prieto \& Garcia, 1997). Bacteria protect themselves from the toxic effects of such compounds by expressing efflux pumps which confer tolerance upon them (Bugg et al., 2000; Hearn et al., 2003; Rojas et al., 2001; van Dyk et al., 2004). An inducible nicotine uptake system has also been shown to exist in Arthrobacter nicotinovorans (Igloi \& Brandsch, 2003), but has not yet been characterized in detail. How A. nicotinovorans controls the intracellular concentration of this potentially toxic compound and of metabolites generated during its breakdown was not known.

The individual steps involved in nicotine degradation by A. nicotinovorans carrying the catabolic plasmid pAO1 are depicted in Fig. 2 (a) (as reviewed by Brandsch, 2006). The gene of the enzyme $\gamma$-N-methylaminobutyrate oxidase (MABO) is part of the purUmabOfolD operon, together with the genes encoding formyltetrahydrofolate deformylase and methylenetetrahydrofolate dehydrogenase-cyclohydrolase (Chiribau et al., 2004). Expression of this operon is 
positively regulated by the transcriptional activator PmfR (Chiribau et al., 2005). MABO demethylates $\gamma$ - $N$-methylaminobutyrate to $\gamma$-aminobutyrate (not shown) (Chiribau et al., 2004). Interestingly, this pathway contains, in addition, an amine oxidase (AO), also under the control of PmfR, which converts $\gamma$-N-methylaminobutyrate into methylamine and succinate semialdehyde (Fig. 2a). Succinate semialdehyde is then oxidized to succinate by a succinate semialdehyde dehydrogenase. The activity of the AO predominates over that of $\mathrm{MABO}$ when the bacteria are grown on citrate medium in the presence of L-nicotine (Chiribau et al., 2006).

Inspection of the pAO1 sequence downstream of the purUmabOfolD operon revealed the presence of two small ORFs, ORF116 and ORF166 (Fig. 1a), with a high degree of similarity to bacterial small multidrug resistance (SMR) pumps. These secondary active antiporters use the proton gradient across the membrane to expel cationic drugs from inside the cell in exchange for at least two protons (Muth \& Schuldiner, 2000; Pornillos et al., 2005). SMR pumps, which are the smallest transporters known, are composed of no more then 105 to 120 amino acids, which form four transmembrane helices (Pornillos et al., 2005). The minimal functional unit of the SMR pump may be formed by a homo- or heterodimer (Jack et al., 2000; Masaoka et al., 2000; Rotem et al., 2001). A variety of cationic drugs are substrates of SMR transporters, which confer upon the bacteria resistance against ethidium, methyl viologen, tetraphenylphosphonium $\left(\mathrm{TPP}^{+}\right)$or tertiary ammonium salts (Morimyo et al., 1992; Yerushalmi et al., 1995).

The localization of two ORFs with similarity to SMR efflux pumps within the gene cluster encoding enzymes of nicotine catabolism stimulated us to investigate their function. Here we show that their genes form a transcriptional unit with the purUmabOfolD operon under the control of PmfR. They encode a two-component efflux pump of the SMR family, responsible for the transport of nicotine breakdown products out of the cell. This pump apparently serves as a metabolic valve for nicotine metabolites and may protect the bacteria from the potentially toxic side effects of these compounds.

(a)

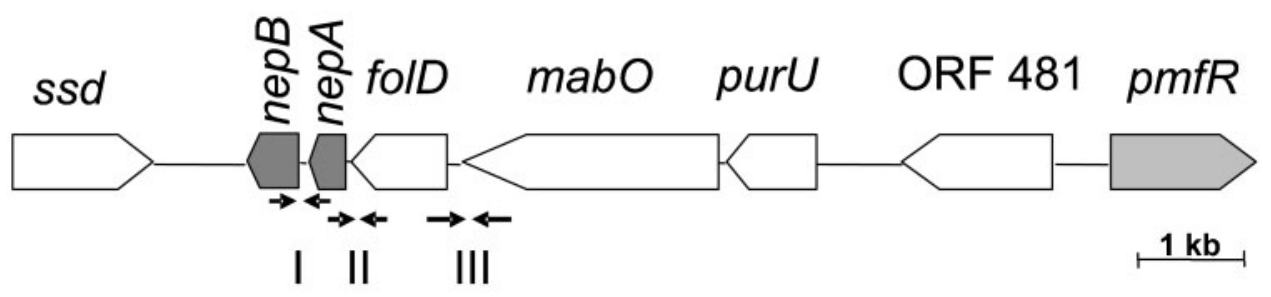

(b)

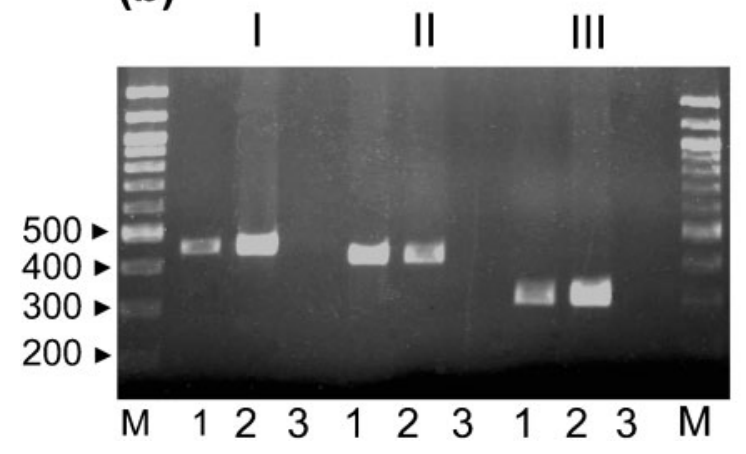

(c)

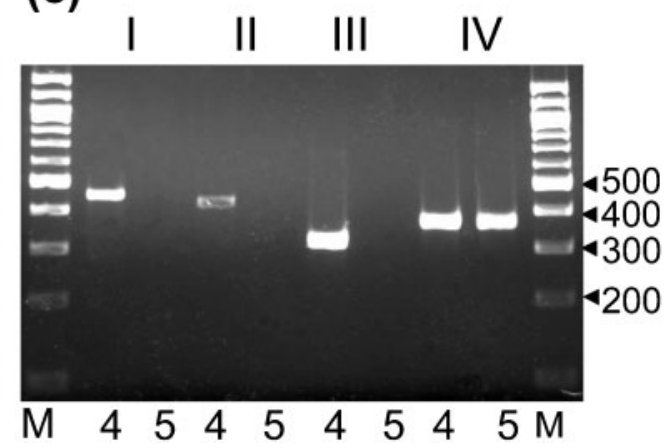

Fig. 1. The pAO1 nepA and nepB genes are part of the PmfR-controlled purUmabOfolD operon and their expression is nicotine-dependent. (a) Schematic representation of the position of the nepAB genes on pAO1 and of primers used in RTPCR reactions. ssd, succinate semialdehyde dehydrogenase; nep, nicotine metabolites efflux pump; fol $D$, methylenetetrahydrofolate dehydrogenase-cyclohydrolase; mabO, $\gamma$-N-methylaminobutyrate oxidase; pur $U$, forrmyltetrahydrofolate deformylase; pmfR, purUmabOfolD transcriptional activator. (b) RT-PCR analysis of transcripts amplified with specific primer pairs: 5 and 6 for I; 7 and 8 for II and 9 and 10 for III (see Table 1). Lanes 1, positive control with A. nicotinovorans cells carrying pAO1 as template; lanes 2, cDNA prepared from nicotine-induced bacteria as template; lanes 3, cDNA prepared from noninduced bacteria as template. (c) RT-PCR with cDNA prepared from the nicotine-induced $A$. nicotinovorans pmfR: $\mathrm{cm} x$ strain and the same primers for I, II and III as indicated in (b). Lanes 4, positive PCR control with A. nicotinovorans cells carrying pAO1 as template; lanes 5, cDNA from nicotine-induced $A$. nicotinovorans pmfR: $c m x$ strain as template. IV, RT-PCR with primer pair 11 and 12 (see Table 1) derived from the $k d h L$ gene, which is not under the control of PmfR, and cDNA prepared from nicotine-induced $A$. nicotinovorans pmfR: $\mathrm{cm} x$ strain as template. M, 100 bp DNA size marker. 

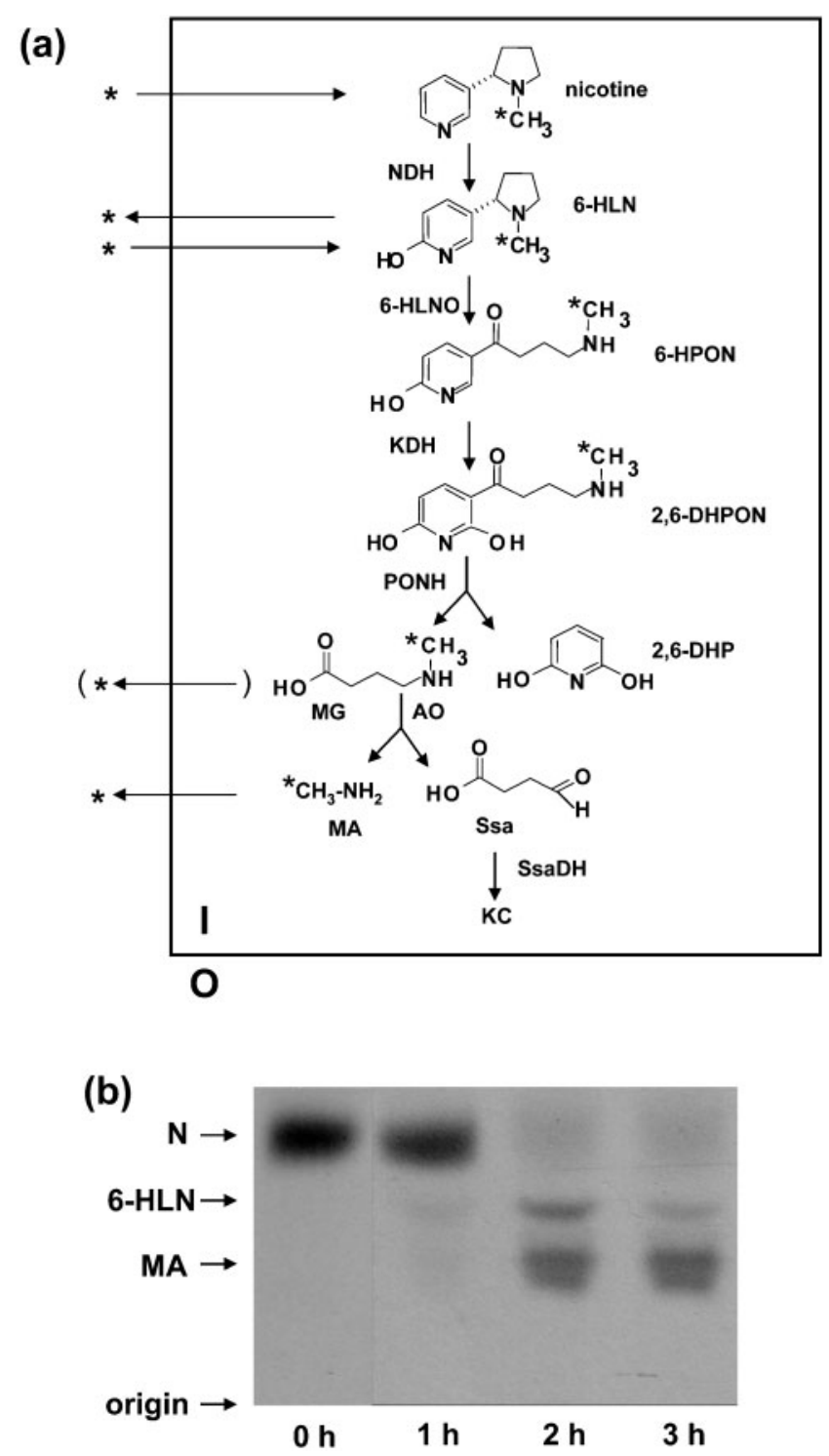

\section{METHODS}

Bacterial strains and growth conditions. A. nicotinovorans wildtype, which carries the catabolic plasmid pAO1, and A. nicotinovorans pmfR: $c m x$, with the gene of the transcriptional activator PmfR disrupted by the insertion of a chloramphenicol resistance cassette (Chiribau et al., 2005), were grown at $30^{\circ} \mathrm{C}$ on citrate medium supplemented with biotin $\left(0.1 \mu \mathrm{g} \mathrm{ml}^{-1}\right)$ and mineral salts solutions (Brühmüller et al., 1975). Escherichia coli XL-1 Blue was used as host for plasmids and was grown at $37^{\circ} \mathrm{C}$ on $\mathrm{LB}$ (lysogeny broth) medium.

Cloning of the pAO1 ORF116 and ORF166 genes. For cloning and expression purposes, the E. coli-A. nicotinovorans shuttle vector pART2 was used (Sandu et al., 2005). It confers kanamycin (Kn) resistance upon $E$. coli (selected with $35 \mu \mathrm{g} \mathrm{Kn} \mathrm{ml}^{-1}$ ) and A. nicotinovorans (selected with $140 \mu \mathrm{g} \mathrm{Kn} \mathrm{ml}^{-1}$ ). The DNA of both ORF116 and ORF166, or ORF116 and ORF166 separately, was amplified by PCR from a pAO1 DNA template with the aid of the primer pairs listed in Table 1, digested with the restriction endonucleases BamHI$\mathrm{Xba \textrm {I }}$ or BamHI-KpnI, respectively, inserted into the multiple cloning
Fig. 2. Nicotine catabolic pathway and efflux of labelled compounds from $A$. nicotinovorans bacteria carrying pAO1 grown in the presence of $\left[{ }^{14} \mathrm{C}\right]$ nicotine. (a) Nicotine catabolic pathway. Site of radioactive label is indicated by an asterisk $\left(^{*}\right)$. The enzymes are: $\mathrm{NDH}$, nicotine dehydrogenase; 6-HLNO, 6-hydroxyL-nicotine oxidase; $\mathrm{KDH}$, ketone dehydrogenase; $\mathrm{PONH}, 2,6$ dihydroxypseudooxynicotine hydrolase; $\mathrm{AO}$, amine oxidase; $\mathrm{SsaDH}$, succinate semialdehyde dehydrogenase. The substrates are: 6-HLN, 6-hydroxy-L-nicotine; 6-HPON, 6-hydroxypseudooxynicotine; 2,6-DHPON, 2,6-dihydroxypseudooxynicotine; MG, $\gamma$-N-methylaminobutyrate; 2,6-DHP, 2,6-dihydroxypyridine; MA, methylamine; Ssa, succinate semialdehyde. KC, Krebs cycle. The bracketed arrow $(\leftarrow)$ indicates that $M G$ was exported only in conditions when it accumulated inside the cells. The frame indicates the cell membrane; I, inside, and $\mathrm{O}$, outside the cell. (b) Efflux of $\left[{ }^{14} \mathrm{C}\right]$ nicotine breakdown products detected by TLC. Overnight cultures of $A$. nicotinovorans grown on citrate medium were supplemented with $100 \mu \mathrm{M}\left[{ }^{14} \mathrm{C}\right]$ nicotine. Samples $(7 \mu \mathrm{l})$ of the medium of each $A$. nicotinovorans culture were taken at the time points indicated, analysed by TLC and the dried plates were autoradiographed on X-Omat AR X-ray film. Arrows indicate the position of labelled compounds as follows: $\mathrm{N}$, nicotine; 6-HLN, 6-hydroxynicotine; MA, methylamine; origin, site of sample application.

site of pART2, ligated and transformed into E. coli XL-1 Blue. Recombinant pART2 plasmid DNA carrying the desired genes was isolated from transformed bacteria, sequenced and transformed by electroporation into A. nicotinovorans strains. Expression of the genes resulted in the synthesis of $\mathrm{C}$-terminally $\mathrm{His}_{8}$-tagged proteins (Sandu et al., 2005).

Determination of intracellular $\left[{ }^{14} \mathrm{C}\right]$ nicotine-derived radioactivity levels. $\mathrm{L}-\left[{ }^{14} \mathrm{C}\right] \mathrm{Nicotine}\left(4.625 \times 10^{7} \mathrm{~Bq} \mathrm{mmol}^{-1}\right)$ (Decker et al., 1961) was used in the experiments as described previously (Igloi \& Brandsch, 2003). The ${ }^{14} \mathrm{C}$-labelled nicotine carries its label at the methyl carbon of the pyrrolidine ring (Fig. 2a). A. nicotinovorans cells were grown overnight in $10 \mathrm{ml}$ citrate medium to stationary phase $\left(\mathrm{OD}_{580}\right.$ 1.0) in the presence of $1.23 \mathrm{mM}$ unlabelled nicotine. The bacteria were pelleted by centrifugation at $3550 \mathrm{~g}$, washed twice with citrate medium, resuspended in the same volume of citrate medium and $\left[{ }^{14} \mathrm{C}\right]$ nicotine was added to $100 \mu \mathrm{M}$. Samples $(1.5 \mathrm{ml})$ were removed at different time points, and the bacteria were pelleted by centrifugation at $18000 \mathrm{~g}$ for $2 \mathrm{~min}$, resuspended in $1 \mathrm{ml}$ double-distilled water and repelleted. The washed bacteria were resuspended in $50 \mu \mathrm{l} 50 \mathrm{mM}$ Tris/ $\mathrm{HCl}(\mathrm{pH} \mathrm{8.0)}, 10 \mathrm{mM}$ EDTA, $1 \mathrm{mg}$ lysozyme $\mathrm{ml}^{-1}$, treated with $100 \mu \mathrm{l} 200 \mathrm{mM} \mathrm{NaOH}-$ $1 \%(\mathrm{w} / \mathrm{v})$ SDS, and neutralized with $75 \mu \mathrm{l}$ of $2 \mathrm{M}$ Tris $/ \mathrm{HCl}$ $(\mathrm{pH}$ 7.0)-0.5 M NaCl. The samples were centrifuged at $18000 \mathrm{~g}$ and the protein concentration in the supernatant was determined by Roti-Quant according to the instructions of the supplier (Roth). Cleared supernatant $(150 \mu \mathrm{l}, 18.5 \mu \mathrm{g}$ protein) was added to $4.5 \mathrm{ml}$ scintillation solution (Roth), and the radioactivity was measured in a Packard liquid scintillation counter for $5 \mathrm{~min}$. The experiments were performed at least three times and the standard deviation was calculated based on these datasets. Scintillation counting revealed that less than $5 \%$ of the radioactivity was present in the pellets of the lysates. The use of filters for quantifying radioactivity proved to be unsuitable for nicotine, because it was not possible to remove non-specifically bound radioactivity from the filters.

To determine the effect of proton-motive force (PMF) inhibitors on the intracellular accumulation of nicotine, $5 \mathrm{mM} \mathrm{2,4-dinitrophenol}$ 
Table 1. Oligonucleotide primers used in this study

\begin{tabular}{|c|c|c|c|}
\hline Primer & Orientation ${ }^{\star}$ & Sequence $\left(5^{\prime}-3^{\prime}\right)$ & $\begin{array}{c}\text { Amplified DNA } \\
\text { fragment (bp) }\end{array}$ \\
\hline \multicolumn{4}{|l|}{ Cloning } \\
\hline 1 & $\mathrm{~F}$ & CCAGGACCACGGATCCTTTGCAGACCAG & 380 перА \\
\hline 2 & $\mathrm{R}$ & GCAGAGTTTGGATCCTTGACCGGCTTG & \\
\hline 3 & $\mathrm{~F}$ & GATAATCGGGGATCCGAGTAGCTATGCAC & 528 перв \\
\hline 4 & $\mathrm{R}$ & CTGTACCCAACTCTAGAGACGTCTTTGCG & \\
\hline 1 & $\mathrm{~F}$ & CCAGGACCACGGATCCTTTGCAGACCAG & 933 перАВ \\
\hline 4 & $\mathrm{R}$ & CTGTACCCAACTCTAGAGACGTCTTTGCG & \\
\hline \multicolumn{4}{|l|}{ RT-PCR } \\
\hline 5 & $\mathrm{~F}$ & GACCAGGTATGGAAGGAGGGC & $457 \mathrm{I}$ \\
\hline 6 & $\mathrm{R}$ & GAATCGCTGTGCAAAAGTTCAAGAC & \\
\hline 7 & $\mathrm{~F}$ & CGTAGGAGTGTCACGGATC & $425 \mathrm{II}$ \\
\hline 8 & $\mathrm{R}$ & GTGCGTAAGCAATTCCGATG & \\
\hline 9 & $\mathrm{~F}$ & CCTTCAATGCTGCAATTCGACCC & 330 III \\
\hline 10 & $\mathrm{R}$ & CCGGTGGACTTCGAGGTCC & \\
\hline 11 & $\mathrm{~F}$ & GGCAACAGACAGGCATGGATAGG & $341 \mathrm{IV}$ \\
\hline 12 & $\mathrm{R}$ & GGATCAACAGCAAGTACAGCAACC & \\
\hline
\end{tabular}

F, forward; R, reverse.

(DNP), $100 \mu \mathrm{M}$ carbonylcyanide $m$-chlorophenylhydrazone (CCCP), $2 \mu \mathrm{M}$ nigericin or $10 \mu \mathrm{M}$ valinomycin was added to the samples together with labelled L-nicotine and the samples were assayed at different time points for intracellular radioactivity. The effective concentration of the inhibitors was established in prior experiments. In order to re-energize bacteria that had been treated for 20 min with nigericin in the presence of labelled nicotine, the bacteria were washed and resuspended in nicotine-free citrate medium plus $2 \mathrm{mM}$ glucose. Samples $(1 \mathrm{ml})$ were taken at different time points, centrifuged, and the level of radioactivity was assayed in the supernatants and in the bacterial pellets as described above.

Thin-layer chromatography. Bacteria were grown on citrate medium overnight at $30^{\circ} \mathrm{C}$ to stationary phase $\left(\mathrm{OD}_{580} 1.0\right)$. L$\left[{ }^{14} \mathrm{C}\right]$ Nicotine $\left(40.7 \times 10^{3} \mathrm{~Bq}\right)$ was added to $10 \mathrm{ml}$ of the culture (which gave a $100 \mu \mathrm{M}$ nicotine concentration), and samples were taken from the growth medium at various time points. Identification of ${ }^{14} \mathrm{C}$-labelled nicotine and nicotine metabolites in the growth medium of bacterial cultures was performed by TLC on Polygram Cel400 plates (Machery-Nagel) loaded with $7 \mu \mathrm{l}$ centrifuged culture medium. The chromatogram was developed with n-butanol/pyridine/acetic acid/ $\mathrm{H}_{2} \mathrm{O}\left(10: 15: 3: 12\right.$; by vol.). The $R_{\mathrm{F}}$ values of unlabelled L-nicotine, 6-hydroxy-L-nicotine, $\gamma$ - $N$-methylaminobutyrate and methylamine that co-migrated on the TLC plates were determined. The front of the methylamine migrating on the TLC showed, under the conditions employed, a higher concentration followed by a less concentrated trail. On the autoradiogram this gives the impression of the spot forming a doublet. The dried TLC plates were exposed to Kodak X-Omat AR film (Sigma) for 7 days and the films were developed in a Curix 60 AGFA machine. The intensity of the bands on the developed films was determined with an Aida ImageAnalyser 3.43 (Raytest).

RNA extraction and reverse transcription PCR (RT-PCR). Total RNA was extracted from A. nicotinovorans cultures and transcribed into cDNA as described previously (Sandu et al., 2003). The cDNA was used at a 1:100 final dilution as template in PCRs with primers specific for the analysed genes (Table 1 and Fig. 1). A. nicotinovorans cells carrying $\mathrm{pAO} 1$ were employed as positive
PCR controls, and RNA was used as a negative PCR control, in order to confirm the absence of contaminating DNA in the RNA preparations.

\section{RESULTS}

\section{Identification of pAO1 ORF116 (nepA) and ORF166 (nepB) gene transcripts as part of the purUmabOfolD operon}

The genes carrying the SMR-like ORFs ORF116 and ORF166 were named, according to their proposed function, nicotine metabolites export pump nepA and nepB, respectively (Fig. 1a). The fact that there are 27 nucleotides between the stop codon of the folD gene and the start codon of nepA and 51 nucleotides between the stop codon of nepA and the start codon of nepB are an indication that these genes may form a transcriptional unit. In order to ascertain this, we performed RT-PCR with primers (Table 1) derived from the $3^{\prime}$ end of one gene and the $5^{\prime}$ end of the next gene as shown in Fig. 1. The cDNA template for the PCR reactions was generated by reverse transcription of RNA extracted from bacteria grown in the absence or presence of nicotine. As shown previously for other pAO1 genes of the nic gene cluster (Chiribau et al., 2005; Sandu et al., 2003), no transcripts of the nерA and nерB genes were detectable in bacteria grown in the absence of nicotine (not shown). In nicotine-grown bacteria, transcripts of these genes were present and the RT-PCR performed with primer pairs derived from the $3^{\prime}$ end of folD of the purUmabOfolD operon and the $5^{\prime}$ end of nepA and with primers derived from the $3^{\prime}$ end of nepA and the $5^{\prime}$ end of nepB indicated that these transcripts form a single RNA molecule (Fig. 1b). 
Since expression of the purUmabOfolD genes is under the control of the transcriptional activator PmfR, transcription of these genes is abolished in a strain in which the $p m f R$ is disrupted by a chloramphenicol resistance cassette (Chiribau et al., 2005). This should apply also to the $n e p A B$ genes if they were part of the same operon. This was indeed the case, as shown in Fig. 1(c).

\section{Intermediates of $\left[{ }^{14} \mathrm{C}\right]$ nicotine catabolism are exported into the culture medium}

Radioactively labelled compounds in the culture medium of A. nicotinovorans grown in the presence of $\left[{ }^{14} \mathrm{C}\right]$ nicotine were analysed by TLC (Fig. 2b). After $2 \mathrm{~h}\left[{ }^{14} \mathrm{C}\right]$ nicotine disappeared from the culture medium and new labelled compounds accumulated. One minor compound comigrated with unlabelled 6-hydroxy-L-nicotine (6-HLN, Fig. 2b) with an $R_{\mathrm{F}}$ value of 0.545 . Another co-migrated with methylamine with a $R_{\mathrm{F}}$ value of 0.433 (MA, Fig. 2b) (Chiribau et al., 2006). Whereas the 6-HLN band disappeared with time from the medium, the MA band, which under these conditions appears to run as a doublet, accumulated and then stayed at a constant level (Fig. 2b). The observed movements of $\left[{ }^{14} \mathrm{C}\right]$ nicotine and $\left[{ }^{14} \mathrm{C}\right]$ nicotine-derived labelled compounds (marked by an asterisk) in and out of bacterial cells are summarized in Fig. 2(a).

\section{Effect of PMF inhibitors on the efflux of $\left[{ }^{14} \mathrm{C}\right]$ nicotine breakdown products from the bacterial cells}

If efflux of ${ }^{14} \mathrm{C}$-labelled compounds was due to the action of an SMR-type pump, PMF inhibitors should affect this process. Bacteria were pre-induced with unlabelled nicotine, washed and resuspended in medium supplemented with $\left[{ }^{14} \mathrm{C}\right]$ nicotine and PMF inhibitors. $\left[{ }^{14} \mathrm{C}\right]$ Nicotine uptake by bacteria with no inhibitors started without a lag period and reached saturation levels within a few minutes (Fig. 3a, asterisks). The presence of the inhibitors did not affect nicotine catabolism during the time-course of the experiment and similar enzyme activity levels were present in treated and untreated bacteria (not shown). However, the PMF inhibitors DNP and CCCP induced a tenfold increase in the accumulation of intracellular radioactivity (Fig. 3a, filled triangles and diamonds). A similar effect was seen with the proton ionophore nigericin. In this case, there was a rapid fivefold accumulation of intracellular radioactivity (Fig. 3a, filled squares) as compared to untreated bacteria (Fig. 3a, asterisks), which after a few minutes reached a (a)

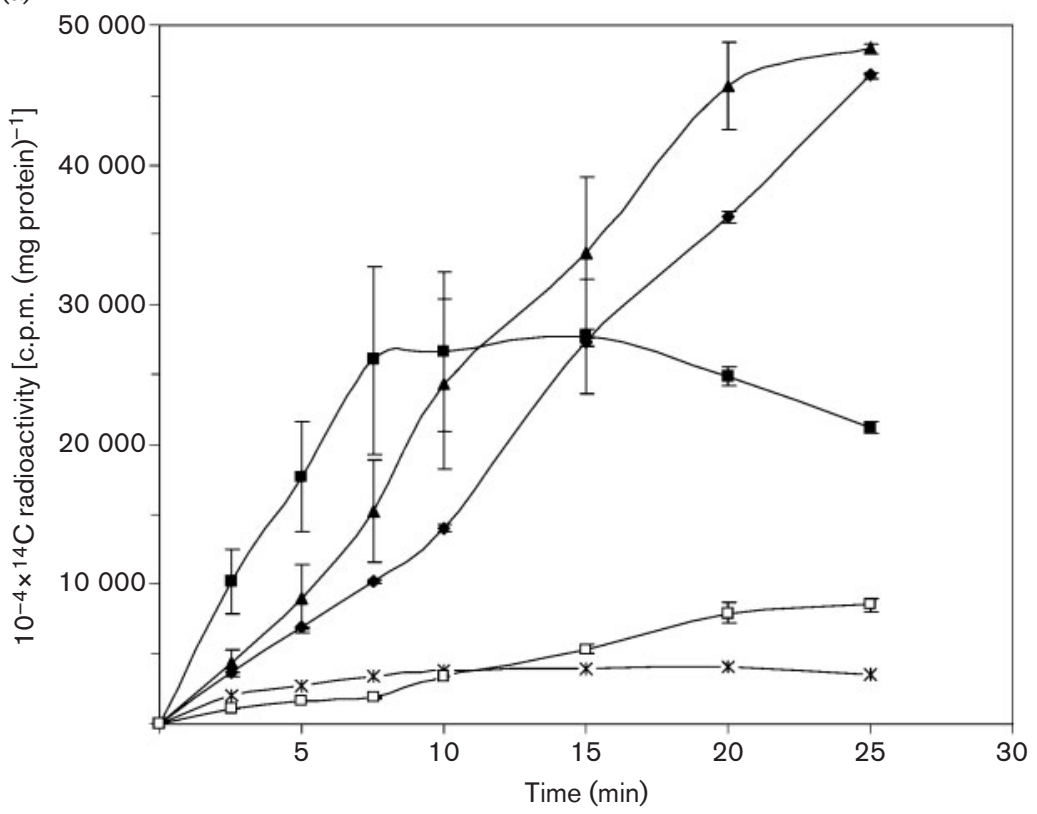

(b)

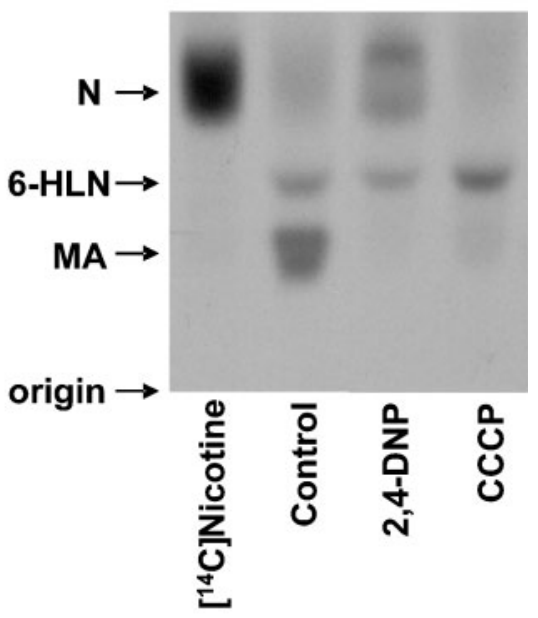

Fig. 3. Effect of PMF inhibitors on the efflux of $\left[{ }^{14} \mathrm{C}\right]$ nicotine-derived radioactivity from $A$. nicotinovorans. Bacteria were grown overnight in the presence of unlabelled nicotine, washed, resuspended in fresh medium supplemented with $\left[{ }^{14} \mathrm{C}\right]$ nicotine and the intracellular $\left[{ }^{14} \mathrm{C}\right]$ nicotine-derived radioactivity was determined as described in Methods. (a) Inhibition of efflux and intracellular accumulation of $\left[{ }^{14} \mathrm{C}\right]$ nicotine-derived radioactivity in the presence of 2,4-DNP (triangles), CCCP (diamonds), nigericin (filled squares), valinomycin (open squares). The control culture without inhibitors is shown as a reference (asterisks). The error bars indicate standard deviations. (b) TLC analysis of ${ }^{14} \mathrm{C}$-labelled compounds in culture media of bacteria incubated for $25 \mathrm{~min}$ in the absence (control) or presence of PMF inhibitors (2,4-DNP and CCCP). The first TLC lane contains labelled nicotine standard. N, nicotine; 6-HLN, 6-hydroxy-L-nicotine; MA, methylamine; origin, site of sample application. 
maximum and then started to decrease. In the presence of the $\mathrm{K}^{+}$ionophore valinomycin the accumulation of intracellular radioactivity was less pronounced (Fig. 3a, open squares). Analysis by TLC of the 25 min culture media of bacteria treated with PMF inhibitors revealed no labelled methylamine (Fig. 3b). Only the small amount of labelled material migrating at the position of 6-HLN was present (Fig. 3b). Apparently, the intracellular accumulation of radioactivity was due to the inhibition of the efflux of radioactive labelled compounds, mainly methylamine, from the bacteria.

In a second set of experiments, bacterial cells were first loaded with $\left[{ }^{14} \mathrm{C}\right]$ nicotine in the presence of the proton ionophore nigericin, then the inhibitor was removed by washing and the bacteria were suspended in glucose medium to regenerate the membrane proton gradient. In the re-energized bacteria, a rapid decrease of the radioactivity that had accumulated inside the nigericin-treated cells was observed (Fig. 4a, open triangles). It was paralleled by an increase in the level of extracellular radioactivity (Fig. 4a, filled triangles). Non-treated bacteria did not show these changes (Fig. 4a, open and filled squares). The increase in extracellular radioactivity correlated with the efflux of methylamine from the nigericin pre-treated and re-energized bacteria as shown in Fig. 4(b).

\section{Efflux of ${ }^{14} \mathrm{C}$-labelled compounds in an A. nicotinovorans pmfR: $\mathrm{cmx}$ background}

RT-PCR analysis of nicotine-induced transcripts of the $n e p A B$ genes revealed that they form one operon with the
purUmabOfolD genes (Fig. 1), controlled by the transcriptional activator PmfR (Chiribau et al., 2005). Thus, in the A. nicotinovorans strain with $p m f R$ disrupted by a chloramphenicol cassette (pmfR:cmx) (Chiribau et al., 2005) expression of the $n e p A B$ genes should be downregulated. In addition, in this strain the gene encoding the amine oxidase responsible for the conversion of $\gamma$-N-methylaminobutyrate into methylamine and succinate semialdehyde (Chiribau et al., 2006) is downregulated, because expression of this gene is also PmfR controlled (C. B. Chiribau \& R. Brandsch, unpublished). In this strain breakdown of $\gamma-\mathrm{N}$-methylaminobutyrate is impaired and the compound should accumulate.

TLC analysis of labelled compounds in the culture medium of $\left[{ }^{14} \mathrm{C}\right]$ nicotine grown bacteria, revealed, as expected, for the pmfR: $c m x$ strain a reduced methylamine level as compared to the wild-type (Fig. $5 \mathrm{a}$, compare lanes 2 and 3 of I and II), and the appearance of a new labelled species (Fig. 5a, II, MG). Densitometric evaluation of the intensity of the methylamine bands in lanes 2 and 3 of groups I and II showed a 2.82 -fold and a 1.56 -fold reduction, respectively. The labelled new species was identified as $\gamma$ - $N$-methylaminobutyrate by its $R_{\mathrm{F}}$ value of 0.477 , which coincided with that of co-migrated unlabelled standard, and by its disappearance from the autoradiogram when treated with MABO, which removes the labelled $\mathrm{CH}_{3}$ group from $\gamma-N$ methylaminobutyrate (not shown). Complementation of the $p m f R: c m x$ strain with nepAB on pART2 should restore efflux of $\gamma-N$-methylaminobutyrate if it was nepAB-dependent. Indeed, in the nepAB-complemented $p m f R: c m x$ strain a strong $\gamma-N$-methylaminobutyrate band was present on the (a)

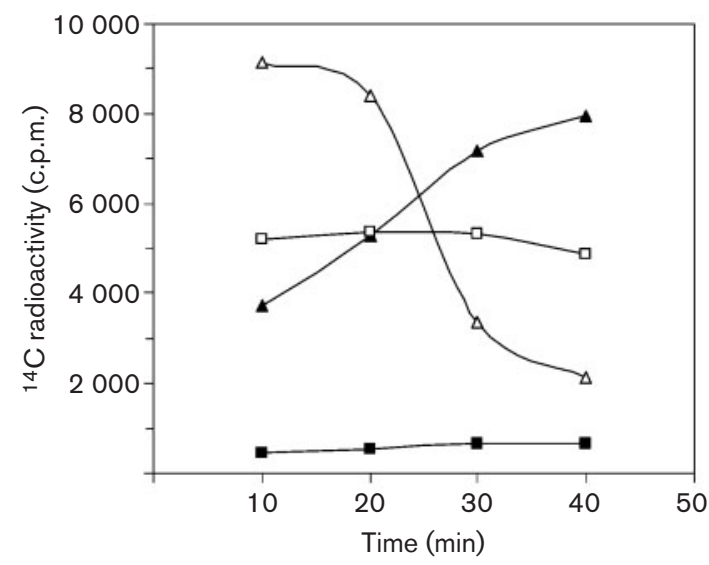

(b)

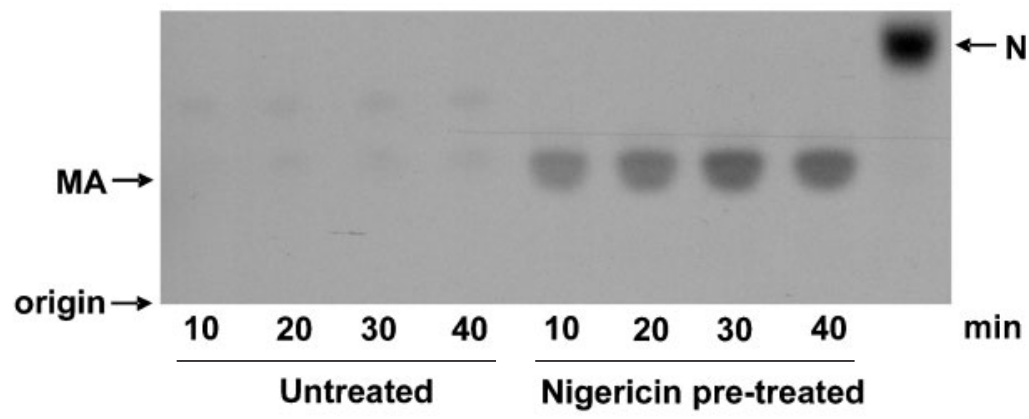

Fig. 4. Efflux of $\left[{ }^{14} \mathrm{C}\right]$ nicotine-derived radioactivity from PMF-inhibited and re-energized $A$. nicotinovorans. (a) Bacteria were treated with nigericin in the presence of $\left[{ }^{14} \mathrm{C}\right]$ nicotine, washed and re-energized with glucose (added at time zero). Samples were taken at the time points indicated and intracellular and extracellular radioactivity was determined using a liquid scintillation counter as described in Methods. Control culture in the absence of nigericin: open squares intra- and closed squares extracellular radioactivity. Nigericin pre-treated culture: open triangles intra- and closed triangles extracellular radioactivity. (b) TLC analysis of labelled compounds in the media of untreated and nigericin pre-treated cultures taken at the same time points as in (a). MA, methylamine; $N$, nicotine; origin, site of sample application. 


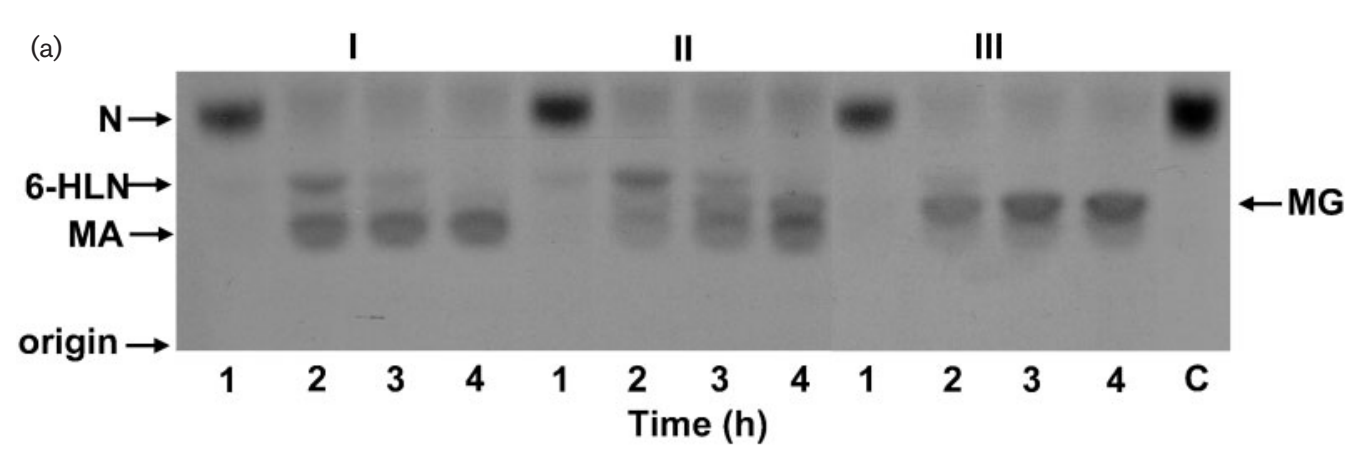

(b)

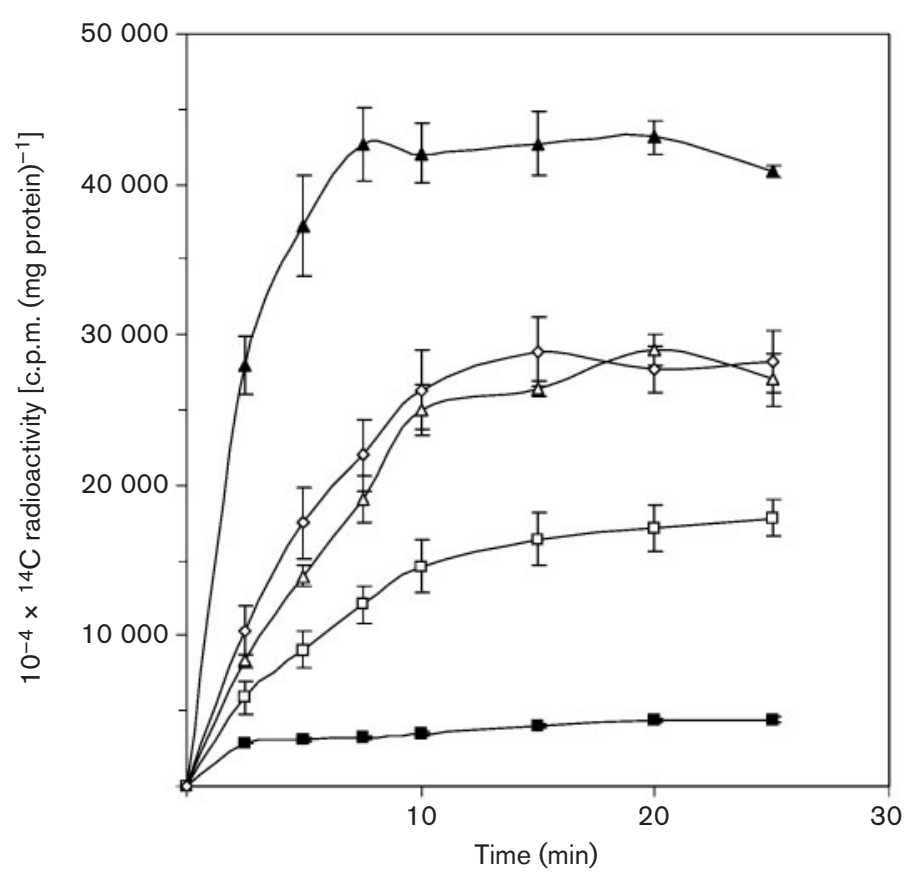

Fig. 5. Comparison of efflux of $\left[{ }^{14} \mathrm{C}\right]$ nicotine-derived labelled compounds from wild-type, pmfR: $c m x$ and pART2nepABcomplemented $A$. nicotinovorans strains. The strains were grown and $\left[{ }^{14} \mathrm{C}\right]$ nicotine-derived compounds were analysed as described in Methods. (a) TLC of ${ }^{14} \mathrm{C}$-labelled compounds in the medium of wild-type (I), pmfR: cmx mutant (II), and pART2nepAB complemented pmfR: $\mathrm{cm} x$ (III), strains. N, nicotine; 6-HLN, 6-hydoxy-L-nicotine; MA, methylamine; MG, $\gamma$ - $N$ methylaminobutyrate; $\mathrm{C}$, labelled nicotine control; origin, site of sample application. (b) Inhibition of efflux and accumulation of intracellular $\left[{ }^{14} \mathrm{C}\right]$ nicotine-derived radioactivity in the $p m f R: \mathrm{cm} x$ strains. A. nicotinovorans pmfR: $\mathrm{cmx}$ (filled triangles) and $A$. nicotinovorans pmfR: $\mathrm{cm} x$ complemented with pART2nepAB (open squares), with pART2nepA (open triangles) and with pART2nepB (open diamonds) were pre-induced with unlabelled nicotine, washed and resuspended in medium with $\left[{ }^{14} \mathrm{C}\right]$ nicotine and the level of intracellular radioactivity was determined as described in Methods. Wild-type levels of intracellular ${ }^{14} \mathrm{C}$-radioactivity are shown by filled squares. The error bars indicate standard deviations.

TLC, but only a small amount of material corresponding to methylamine (Fig. 5a, III) was observed.

When bacteria, pre-induced with unlabelled nicotine, were tested in $\left[{ }^{14} \mathrm{C}\right]$ nicotine uptake assays, intracellular radioactivity rapidly accumulated to a high level in the $p m f R: c m x$ strain with downregulated nepAB (Fig. 5b, filled triangles). Complementation of the strain with nepAB induced a strong efflux of intracellular radioactivity (Fig. 5b, compare filled triangles with open squares), which however did not reach wild-type levels (Fig. 5b filled squares). Complementation of the strain with nepA or nepB individually produced an efflux of intracellular radioactivity, which however was less pronounced than with nepAB (Fig. 5b). Expression of перAB in strains transformed with pART2nepAB was confirmed by the identification of nepAB transcripts by RT-PCR and of NepB-His 8 protein on Western blots of bacterial membranes developed with anti-His-specific antibody (not shown).

\section{DISCUSSION}

In agreement with the observation that $A$. nicotinovorans is unable to use methylamine as a growth substrate (P. Ganas 
\& R. Brandsch, unpublished), this metabolic end-product of nicotine catabolism was shown here to be exported from the cell. Our investigation suggests that the pAO1 NepAB efflux pump represents the metabolic valve responsible for this process. This is, to our knowledge, the first time that an SMR pump has been shown to be involved in the control of the intracellular concentration of components of a metabolic pathway. A first hint as to this role of NepAB came from the finding that the nерAB genes are co-transcribed in a nicotine-dependent fashion with the $\mathrm{pAO} 1$ purUmabOfolD operon. NepAB may have an essential function in the regulation of the intracellular levels of metabolites.

This conclusion was supported by the TLC analysis of $\left[{ }^{14} \mathrm{C}\right]$ nicotine catabolites exported from the bacterial cells into the growth medium and identified as 6-HLN and methylamine. The appearance of 6-HLN in the medium of A. nicotinovorans grown on nicotine has been reported earlier (Decker et al., 1961; Hochstein \& Rittenberg, 1959) and has been used to isolate this compound for chemical syntheses (Roduit et al., 1997). 2,6-Dihydroxypseudooxynicotine (2,6-DHPON) seems to be rapidly turned over by pseudooxynicotine hydrolase (PONH) into 2,6-DHP and $\gamma$-N-methylaminobutyrate and has not been shown to accumulate (Gherna et al., 1965). The time-dependent disappearance of 6-HLN from the culture medium suggested that it re-enters the cells and is turned over by the bacteria. In contrast, methylamine behaved like a metabolic end-product and showed a continuous efflux from the bacteria and accumulated in the growth medium.

Support for the involvement of an SMR efflux pump in nicotine metabolism came from the effect of PMF inhibitors on the efflux of $\left[{ }^{14} \mathrm{C}\right]$ nicotine-derived catabolic intermediates. Members of the SMR pumps family make use of the $\mathrm{H}^{+}$gradient across the cell membrane to export cationic drugs from bacterial cells in an anti-port mechanism (Paulsen et al., 1996, Putman et al., 2000). The strong increase in intracellular and the strong decrease in extracellular radioactivity in the presence of 2,4-DNP and CCCP pointed to the possibility that efflux of the nicotinederived compounds into the medium was due to the action of such a pump. CCCP and 2,4-DNP inhibit both components of the PMF, the proton gradient $\Delta \mathrm{pH}$ and the electrochemical gradient $\Delta \Psi$. Both components may contribute to the inhibition of the efflux of labelled nicotine breakdown products. However, the very much reduced effect of valinomycin on the accumulation of intracellular radioactivity suggests that the $\Delta \mathrm{pH}$ is the main driving force in the efflux of methylamine. Nigericin, a $\Delta \mathrm{pH}$ inhibitor, showed, at a concentration of $2 \mu \mathrm{M}$, a clear inhibition of the efflux of labelled material from bacteria. The lower level of accumulation of intracellular radioactivity and its slow decrease over time indicated that the efflux pump was not completely inhibited. Support for the involvement of an SMR pump came also from the finding that efflux of labelled compounds from bacteria was resumed following regeneration of the PMF.
Efforts to disrupt the nерAB genes by insertion of a chloramphenicol cassette were unsuccessful. However, strong support for NepAB being the two-component efflux pump for nicotine degradation products came from the $p m f R: \mathrm{cm} x$ strain. Since the nepAB genes form one operon with the purUmabOfoldD genes and expression of this operon is regulated by the transcriptional activator $p m f R$, expression of the nepAB genes in a pmfR-disrupted strain (Chiribau et al., 2005) should be downregulated. This was indeed the case, as shown by transcriptional analysis by RT-PCR. In the $p m f R$-disrupted strain, there was a strong accumulation of intracellular radioactivity, analogous to the effect of PMF inhibitors. We take this as a clear indication that nepAB are the genes of the pump responsible for the efflux of nicotine catabolites, in particular methylamine. In the $p m f R$-disrupted strain the turnover of $\gamma$ - $N$-methylaminobutyrate should be impaired because the genes encoding $\mathrm{MABO}$ and $\mathrm{AO}$ are also downregulated. The identification of this compound in the culture medium of $\left[{ }^{14} \mathrm{C}\right]$ nicotine grown pmfR: $c m x$ strain points exactly to this fact. This shows that the efflux pump is also capable of exporting $\gamma$-N-methylaminobutyrate. Thus NepAB indeed works as a metabolic valve in nicotine catabolism, able to expel metabolic intermediates when they accumulate. In the $p m f R: c m x$ strain production and efflux of methylamine were not completely abolished, which may be explained by a residual low-level expression of the PmfR-regulated genes in the absence of the activator. Alternatively it can not be excluded that another transport system may be responsible for this residual MA export activity.

When additional copies of nерAB were introduced on pART2 into the pmfR: $c m x$ strain the intracellular level of radioactivity that accumulated in the $p m f R: c m x$ strain was reduced by $50 \%$. We interpret this finding as being due to an increased amount of NepAB pumps in the bacterial membrane. Both components, nepA and nep $B$, were required to reach the full effect, supporting the conclusion that $\mathrm{NepAB}$ is a two-component efflux pump for nicotine catabolites.

The importance of efflux pumps for tolerance to toxic organic compounds has been documented during recent years for several bacterial systems (Bugg et al., 2000; Hearn et al., 2003; Rojas et al., 2001; van Dyk et al., 2004). However, there was no clear increase in resistance of $E$. coli and $A$. nicotinovorans transformed with pART2nepAB against compounds like ethidium, methyl viologen or $\mathrm{TPP}^{+}$, compounds shown to be substrates for other members of the SMR pumps.

The efflux pump PbuE of the major facilitator superfamily (MFS) has been demonstrated to play an important role in purine metabolism in Bacillus subtilis by regulating the purine base pool size (Nygaard \& Saxild, 2005). NepAB is to our knowledge the first SMR type efflux pump shown to act as a metabolic valve in a bacterial catabolic pathway. Considering the chemical heterogeneity of the exported nicotine catabolites, a pump of the SMR family, which is 


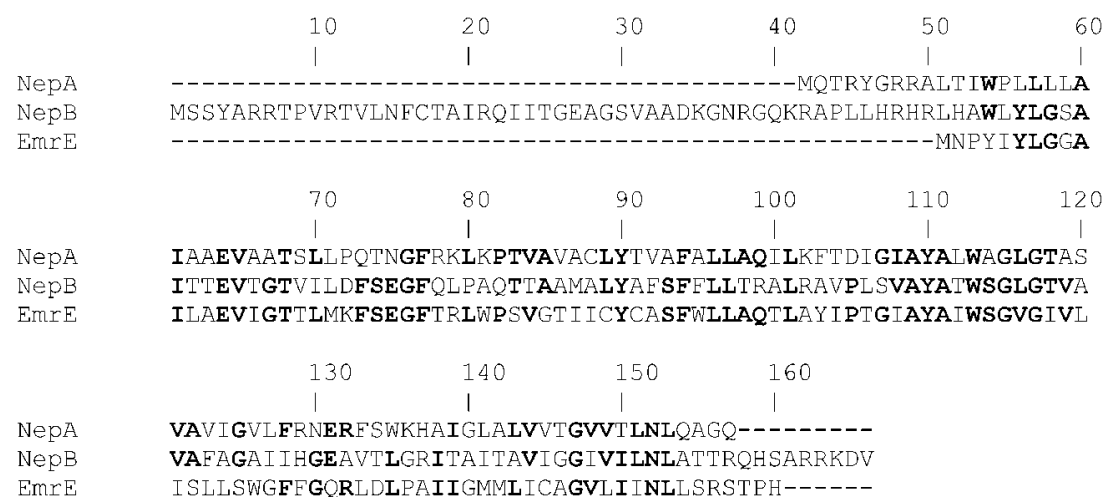

Fig. 6. Alignment of the amino acid sequences of $A$. nicotinovorans $\mathrm{NepA}$ and $\mathrm{NepB}$, and E. coli EmrE. Conserved residues are indicated in bold. characterized by the ability to transport a wide variety of structurally different substrates, seems very well suited to serve this purpose.

A search performed at the SIB BLAST Network Service of the Swiss Institute of Bioinformatics with the amino acid sequences of NepA and NepB indicated a high degree of similarity to EmrE of E. coli (38\% with NepA; $34 \%$ with NepB), a prototype SMR pump (Schuldiner et al., 2001; Yerushalmi et al., 1995) of known structure (Ma \& Chang, 2004; Pornillos et al., 2005), which confers resistance to ethidium, methyl viologen and $\mathrm{TPP}^{+}$. Of the amino acid residues of EmrE shown by site-directed mutagenesis and confirmed by the X-ray structure to be essential for activity, most are conserved in NepA and NepB (Fig. 6). Thus, the EmrE glutamate residue E14 of each monomer is located at the interface of the dimer and binds the positively charged substrate (Gutman et al., 2003; Muth \& Schuldiner, 2000). In NepA the corresponding glutamate

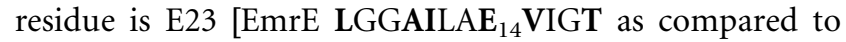
NepA LLLAIAAE ${ }_{23}$ VAAT (conserved residues in bold)]. In NepB it is glutamate residue E64 [EmrE LGGAILAE $_{14}$ VIGT

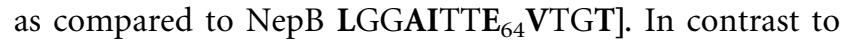
NepA, a polypeptide of 116 amino acids with a predicted molecular mass of $12439.8 \mathrm{Da}$, NepB, a polypeptide of 166 amino acids and a predicted molecular mass of 17749.6 $\mathrm{Da}$, when aligned with the amino acid sequence of EmrE or of other SMR pumps, exhibits an unusually large hydrophilic N-terminal extension of approximately 50 amino acids (Fig. 6). The hydrophilic N-terminal domain of NepB is also unusual with respect to the B subunits of other SMR two-component efflux pumps, like EbrAB of B. subtilis (Masaoka et al., 2000) and YdgEF of E. coli (Nishino \& Yamaguchi, 2001), which do not have this $\mathrm{N}$-terminal extension (not shown). Therefore NepB seems to represent a new subtype of SMR pumps. The two EmrE monomers are positioned in an inverted orientation in the membrane and form an anti-parallel asymmetric dimer (Gottschalk et al., 2004, Pornillos et al., 2005, UbarretxenaBelandia et al., 2003). This particular arrangement may determine a unidirectional transport. NepA and NepB are predicted to contain four transmembrane helices (Daley et al., 2005, Granseth et al., 2005), typical for SMR pumps, and to exhibit an inverted orientation of the two subunits in the membrane. This agrees with the complementation results of the A. nicotinovorans pmfR: $c m x$ strain with pART nepA or pART2nep $B$ individually and in combination, which suggest that nep $A B$ is a two-component efflux pump.

\section{ACKNOWLEDGEMENTS}

This work was supported by a grant of the Deutsche Forschungsgemeinschaft to R. B.

\section{REFERENCES}

Brandsch, R. (2006). Microbiology and biochemistry of nicotine degradation. Appl Microbiol Biotechnol 69, 493-498.

Brühmüller, M. A., Schimz, A., Messmer, L. \& Decker, K. (1975). Covalently bound FAD in D-6-hydroxynicotine oxidase. J Biol Chem 250, 7747-7751.

Bugg, T., Foght, J. M., Pickard, M. A. \& Gray, M. R. (2000). Uptake and active efflux of polycyclic aromatic hydrocarbons by Pseudomonas fluorescens LP6a. Appl Environ Microbiol 66, 5387-5392.

Chiribau, C.-B., Sandu, C., Fraaije, M., Schiltz, E. \& Brandsch, R. (2004). A novel $\gamma$-N-methylaminobutyrate demethylating oxidase involved in catabolism of the tobacco alkaloid nicotine by Arthrobacter nicotinovorans pAO1. Eur J Biochem 271, 4677-4684.

Chiribau, C.-B., Sandu, C., Igloi, G. L. \& Brandsch, R. (2005). Characterization of PmfR, the transcriptional activator of the pAO1borne purU-mabO-folD operon of Arthrobacter nicotinovorans. J Bacteriol 187, 3062-3070.

Chiribau, C.-B., Mihasan, M., Ganas, P., Igloi, G. L., Artenie, V. \& Brandsch, R. (2006). Final steps in the catabolism of nicotine. Deamination versus demethylation of $\gamma$-N-methylaminobutyrate. FEBS J 273, 1528-1536.

Daley, D. O., Rapp, M., Granseth, E., Melén, K., Drew, D. \& von Heijne, G. (2005). Global topology analysis of the Escherichia coli inner membrane proteome. Science 308, 1321-1323.

Decker, K., Eberwein, H., Gries, F. A. \& Brühmüller, M. (1961). Über den Abbau des Nicotins durch Bakterienenzyme. IV. L-6-Hydroxynicotine als erstes Zwischenprodukt. Biochem $Z$ 334, 227-244.

Gherna, R. L., Richardson, S. H. \& Rittenberg, S. C. (1965). The bacterial oxidation of nicotine. VI. The metabolism of 2,6dihydroxypseudooxynicotine. J Biol Chem 240, 3669-3674.

Gottschalk, K.-E., Soskine, M., Schuldiner, S. \& Kessler, H. (2004). A structural model of EmrE, a multi-drug transporter from Escherichia coli. Biophys J 86, 3335-3348. 
Granseth, E., Daley, D. O., Rapp, M., Melén, K. \& von Heijne, G. (2005). Experimentally constrained topology models for 51,208 bacterial inner membrane proteins. J Mol Biol 352, 489-494.

Gutman, N., Steiner-Mordoch, S. \& Schuldiner, S. (2003). An amino acid cluster around the essential Glu-14 is part of the substrate- and proton-binding domain of EmrE, a multidrug transporter from Escherichia coli. J Biol Chem 278, 16082-16087.

Hearn, E. M., Dennis, J. J., Gray, M. R. \& Foght, J. M. (2003). Identification and characterization of the emhABC efflux system for polycyclic aromatic hydrocarbons in Pseudomonas fluorescens cLP6a. J Bacteriol 185, 6233-6240.

Hochstein, L. I. \& Rittenberg, C. S. (1959). The bacterial oxidation of nicotine. II. The isolation of the first product and its identification as (L)-6-hydroxynicotine. J Biol Chem 234, 156-162.

Hukkanen, J., Jacob, P. \& Benowitz, N. L. (2005). Metabolism and disposition kinetics of nicotine. Pharmacol Rev 57, 79-115.

Igloi, G. L. \& Brandsch, R. (2003). Sequence of the 165-kilobase catabolic plasmid pAO1 from Arthrobacter nicotinovorans and identification of a pAO1-dependent nicotine uptake system. J Bacteriol 185, 1976-1989.

Jack, D. L., Storms, M. L., Tchieu, J. H., Paulsen, I. T. \& Saier, M. H., Jr (2000). A broad-specificity multidrug efflux pump requiring a pair of homologous SMR-type proteins. J Bacteriol 182, 2311-2313.

Lee, G., Dallas, S., Hong, M. \& Bendayan, R. (2001). Drug transport in the central nervous system: brain barriers and brain parenchyma considerations. Pharmacol Rev 53, 569-596.

Leveau, J. H. J., Zehnder, A. J. B. \& van der Meer, J. R. (1998). The $t f d K$ gene product facilitates the uptake of 2,4-dichlorophenoxyacetate by Ralstonia eutropha JMP134 (pJP4). J Bacteriol 180, 2237-2243.

Locher, H. H., Poolman, B., Cook, A. M. \& Konings, W. N. (1993). Uptake of 4-toluene sulfonate by Comamonas testosteroni T-2. J Bacteriol 175, 1075-1080.

Ma, C. \& Chang, G. (2004). Structure of the multidrug resistance efflux transporter EmrE from Escherichia coli. Proc Natl Acad Sci U S A 101, 2852-2857.

Masaoka, Y., Ueno, Y., Morita, Y., Kuroda, T., Mizushima, T. \& Tsuchiya, T. (2000). A two-component multidrug efflux pump, EbrAB, in Bacillus subtilis. J Bacteriol 182, 307-310.

Morimyo, M., Hongo, E., Hama-Inaba, H. \& Machida, I. (1992). Cloning and characterization of the $m v r C$ gene of Escherichia coli $\mathrm{K}-12$ which confers resistance against methyl viologen toxicity. Nucleic Acids Res 20, 3159-3165.

Muth, T. R. \& Schuldiner, S. (2000). A membrane-embedded glutamate is required for ligand binding to the multidrug transporter EmrE. EMBO J 19, 234-240.

Nichols, N. N. \& Harwood, C. S. (1997). PcaK, a high-affinity permease for the aromatic compounds 4-hydroxybenzoate and protocatechuate from Pseudomonas putida. J Bacteriol 179, 50565061.

Nishino, K. \& Yamaguchi, A. (2001). Analysis of a complete library of putative drug transporter genes in Escherichia coli. J Bacteriol 183, $5803-5812$.
Nygaard, P. \& Saxild, H. H. (2005). The purine efflux pump PbuE in Bacillus subtilis modulates expression of the PurR and G-box (XptR) regulon by adjusting the purine base pool size. I Bacteriol 187, 791-794.

Paulsen, I. T., Brown, M. H. \& Skurray, R. A. (1996). Protondependent multidrug efflux systems. Microbiol Rev 60, 575-608.

Pornillos, O., Chen, Y.-J., Chen, A. P. \& Chang, G. (2005). X-ray structure of the EmrE multidrug transporter in complex with a substrate. Science 310, 1950-1953.

Prieto, M. A. \& Garcia, J. L. (1997). Identification of the 4hydroxyphenylacetate transporter gene of Escherichia coli W: construction of a highly sensitive cellular biosensor. FEBS Lett 414, 293-297.

Putman, M., van Veen, H. W. \& Konings, W. N. (2000). Molecular properties of bacterial multidrug transporters. Microbiol Mol Biol Rev 64, 672-693.

Roduit, J.-P., Wellig, A. \& Kiener, A. (1997). Renewable functionalized pyridines derived from microbial metabolites of the alkaloid $(S)$ nicotine. Heterocycles 45, 1687-1702.

Rojas, A., Duque, E., Mosqueda, G., Golden, G., Hurtado, A., Ramos, J. L. \& Segura, A. (2001). Three efflux pumps are required to provide efficient tolerance to toluene in Pseudomonas putida DOT-T1E. J Bacteriol 183, 3967-3973.

Rotem, D., Sal-man, N. \& Schuldiner, S. (2001). In vitro monomer swapping in EmrE, a multidrug transporter from Escherichia coli, reveals that the oligomer is the functional unit. J Biol Chem 276, 48243-48249.

Sandu, C., Chiribau, C.-B. \& Brandsch, R. (2003). Characterization of HdnoR, the transcriptional repressor of the 6-hydroxy-D-nicotine oxidase gene of Arthrobacter nicotinovorans pAO1, and its DNAbinding activity in response to L- and D-nicotine derivatives. $J$ Biol Chem 278, 51307-51315.

Sandu, C., Chiribau, C.-B., Sachelaru, P. \& Brandsch, R. (2005). Plasmids for nicotine-dependent and independent gene expression in Arthrobacter nicotinovorans and other Arthrobacter species. Appl Environ Microbiol 71, 8920-8924.

Schuldiner, S., Granot, D., Mordoch, S. S., Nino, S., Rotem, D., Soskin, M., Tate, C. G. \& Yerushalmi, H. (2001). Small is mighty: EmrE, a multidrug transporter as an experimental paradigm. News Physiol Sci 16, 130-134.

Ubarretxena-Belandia, I., Baldwin, J. M., Schuldiner, S. \& Tate, C. G. (2003). Three-dimensional structure of the bacterial multidrug transporter EmrE shows it is an asymmetric homodimer. EMBO J 22, 6175-6181.

van Dyk, T. K., Templeton, L. J., Cantera, K. A., Sharpe, P. L. \& Sariaslani, F. S. (2004). Characterization of the Escherichia coli AaeAB efflux pump: a metabolic valve? J Bacteriol 186, 7196-7204. Yerushalmi, H., Lebendiker, M. \& Schuldiner, S. (1995). EmrE, an Escherichia coli $12-\mathrm{kDa}$ multidrug transporter, exchanges toxic cations and $\mathrm{H}^{+}$and is soluble in organic solvents. J Biol Chem 270, 6856-6863.

Edited by: J. M. Becker 